\title{
Exclusiones y discriminaciones hacia las identidades trans en educación afectivo-sexual
}

\author{
Alejandro Granero Andújar \\ Universidad de Almería, Almería, España \\ mail: aga848Qual.es \\ ORCID: https://orcid.org/0000-0003-2697-0964
}

\section{RESUMEN}

Este trabajo pretende conocer en profundidad la presencia y el tratamiento que reciben las identidades trans en prácticas de educación afectivo-sexual. Como eje de indagación, se tomaron los talleres implementados en el marco del Programa Forma Joven en dos Institutos de Educación Secundaria de la ciudad de Almería. Para ello, se ha llevado a cabo una investigación de corte cualitativo, tomando el estudio de caso múltiple como estrategia de investigación. Como técnicas de recogida de datos fueron empleadas la entrevista semiestructurada en profundidad, la observación no participante y el análisis de documentos. Los resultados muestran el silenciamiento y el tratamiento superficial de este colectivo, unido a la transmisión de concepciones excluyentes y valores discriminatorios, tales como el ciscentrismo, el binarismo de género y la monosexualidad. Se concluye que dichas prácticas contribuyen a la reproducción y legitimación de una visión estigmatizada de las identidades trans que conlleva a que estas sean percibidas como anormales, excepcionales y tabú por no encontrarse en la cotidianeidad académica, así como por los mensajes excluyentes transmitidos.

Palabras Clave: educación sexual; identidades trans; estudio de caso múltiple; jóvenes; LGTB.

\section{Exclusion and discrimination against transgender identities in affective-sexual education}

\section{ABSTRACT}

This article aims to attain an in-depth knowledge regarding the presence and treatment that transgender identities receive in affectivesexual education practices. The research focuses on the workshops implemented under the Forma Joven Program which were conducted at two Secondary Education Institutes in the city of Almería. To this end, qualitative research was carried out, taking the multiple case study as a research strategy. The data collection techniques used were semi-structured in-depth interview, non-participatory observation, and document analysis. The results show the silencing and superficial treatment of this collective, coupled with the transmission of exclusionary conceptions and discriminatory values, such as ciscentrism, gender binarism, and monosexuality. It is concluded that such practices, along with the exclusionary messages transmitted, contribute to the reproduction and legitimization of a stigmatized view of transgender identities that leads to them being perceived as abnormal, exceptional, and taboo, since they are not found in academic daily life.

Keywords: sexual education; transgender; multiple-case study; young people; LGBT.

ISSN: 0210-2773

DOI: https://doi.org/10.17811/rifie.50.4.2021.833-840 


\section{Introducción}

Este trabajo presenta las reflexiones y resultados de una investigación desarrollada durante seis años, cuyo objetivo se ha centrado en alcanzar conocimientos y comprensiones acerca de la posible reproducción de discriminaciones e invisibilizaciones hacia las personas trans en el ámbito de la educación afectivo-sexual.

Consideramos que dicho campo educativo se convierte en el espacio de mayor idoneidad a la hora de atender a las identidades y corporalidades Lesbianas, Gays, Trans, Bisexuales e Intersexuales (en adelante, LGTBI) en los contextos escolares, dada la estrecha relación que guardan sus contenidos con este colectivo. Por ello, el trabajo de investigación que aquí se presenta se ha centrado en el estudio en profundidad de los talleres de educación afectivo-sexual implementados en el marco del Programa Forma Joven en dos Institutos de Educación Secundaria de la provincia de Almería (España).

La elección de dicho programa vino motivada por ser el único espacio educativo donde se aborda la educación afectivo-sexual de manera institucionalizada en la Comunidad Autónoma de Andalucía, siendo este implementado desde el curso 2001/02 por las Consejerías de la Junta de Andalucía de Salud, de Educación, de Igualdad y Políticas Sociales y de Gobernación. Asimismo, en su área de intervención Sexualidad y Relaciones Igualitarias queda recogido el abordaje del colectivo LGTBI, según la propia web del Programa.

La revisión de la literatura en las principales bases de datos científicas (SCOPUS, DIALNET, TESEO, ISOC, ERIC, EDUCATION SOURCE, REDIB, OAISTER, REDALYC y Web of Science) ha manifestado una escasez de trabajos científicos sobre las identidades trans en el campo de la educación afectivo-sexual. Las investigaciones que recogen experiencias sobre ello son muy reducidas en el ámbito internacional, mientras que no se han localizado trabajos científicos en el contexto español. La mayoría de los aportes analizados identificaron las prácticas de educación afectivo-sexual como espacios donde las identidades trans quedan desatendidas por no ajustarse a los parámetros hegemónicos de la categorización biológica y binaria de género (Jones et al., 2016), generando sentimientos hostiles en el alumnado trans hacia este campo educativo a la vez que no son atendidas sus necesidades formativas (Estes, 2016; Hobaica y Kwon, 2017; Rivers y Noret, 2008; Smith et al., 2008).

Más detalladamente, De Palma y Francis (2014), a partir del estudio de las percepciones del profesorado, mostraron la educación sexual como un dispositivo de conformación de masculinidades y feminidades, las cuales se entendían como naturales en función de la categorización biológica de sexo. Los datos aportados por Quaresma y Ulloa (2011) visibilizaron las actitudes reticentes de docentes para abordar las identidades trans en prácticas de educación afectivo-sexual. Morton (2010) identificó cómo se hacía notable la falta de concienciación sobre diversidad sexo-genérica entre el profesorado a partir de sus actitudes y mensajes. En menor medida, también es posible hallar estudios sobre prácticas de educación afectivo-sexual inclusivas con las identidades trans (Ekstrand et al., 2011; Grotz et al., 2016), señalando sus potencialidades en el desarrollo de actitudes y valores igualitarios entre el alumnado (Chi et al., 2015).

\section{Revisión literaria y objetivos}

Para conceptualizar qué son las identidades trans, es necesario recurrir a la relación entre categorización de sexo (biológico) y de género (autodeterminado), derivándose las posibilidades de identidad de género a dos opciones estructurales. De una parte, podemos hablar de identidades cis cuando el género sentido por la persona coincide con el asignado en el momento de su nacimiento en base a criterios biológicos. Criterios biológicos que han sido cuestionados desde un enfoque post-estructuralista dada su construcción social (Butler, 2007; Foucault, 2012).

De otra parte, hablamos de identidades trans para hacer referencia a las personas cuyo género sentido no se corresponde con el asignado en el nacimiento. En la globalidad de este concepto se encuentran la transexualidad y el transgenerismo. La primera hace mención a las personas cuyo género autodeterminado difiere al asignado en su nacimiento, adecuando así sus características fisiológicas a través de procesos hormonales y/o quirúrgicos, o teniendo intención de hacerlo, para integrarse en una de las dos categorías binarias de género (Lamas, 2009). Por su parte, transgenerismo representa al conjunto de personas que transicionan a formas no binarias de género, es decir más allá de hombre y mujer (Balza, 2009).

El término identidades trans también puede integrar el travestismo. En el contexto europeo, este agrupa a quienes, de manera puntual, utilizan ropa y complementos que tradicionalmente han sido considerados de un género diferente al suyo, existiendo correspondencia entre su género y el asignado en el nacimiento (Lamas, 2009). No obstante, en contextos latinoamericanos es empleado como sinónimo de transexualidad y transgenerismo. A pesar de estas diferencias culturales, cabe aclarar que en este trabajo se parte de la aceptación del término identidades trans únicamente para hacer referencia a las identidades en las que ha existido o hay intención de llevar a cabo un proceso de transición de género.

En las diferentes culturas, los valores y concepciones hegemónicas ejercen mecanismos de control y dominación en todas las dimensiones que ocupan nuestro sentido personal, extendiéndose también a la esfera de la sexualidad. En palabras de Collignon (2011):

La sexualidad es finalmente una construcción social que se encuentra determinada por las condiciones sociales, culturales, históricas, políticas y económicas de la sociedad a la que pertenece. (p. 137).

Desde el campo de la filosofía se ha profundizado en este sentido sociocultural restrictivo de la sexualidad. Foucault (2012), mediante el término biopoder, señaló que nuestras formas de entender el mundo, comportarnos, desear y percibir los cuerpos están determinadas por las formas de construcción de la cultura dentro de un contexto social concreto. Así, el biopoder actúa mediante: en primer lugar, la estandarización y optimización de las funciones y posibilidades corporales por parte de las ciencias del saber (medicina, psicología, sociología...); en segundo lugar, el control disciplinario del género y todo lo que en el sistema dominante se construye a su alrededor (deseo afectivo-sexual, comportamientos, roles, tareas, gestos...). Preciado (2003) introdujo el concepto de sexopolítica como "una de las formas dominantes de la acción biopolítica en el capitalismo contemporáneo" (p. 157). Con él mostraba cómo el sexo y todo lo que rodea a este (órganos sexuales, prácticas afectivo-sexuales, identidades de género y códigos de masculinidad y feminidad) son moldeados en los límites de los modelos normativos mediante los discursos, convirtiéndose el poder en un agente de control de las vidas de las personas. Butler (2007), empleando el término matriz heterosexual, expuso el modo en que los cuerpos, el género y los deseos son conformados según los parámetros de la normatividad social mediante lo que denomina como performatividad, esto es la construcción de las subjetividades a través de la repetición de rituales y patrones tradicionales asociados a los géneros. 
Específicamente, la literatura del ámbito científico-académico y las aportaciones de los movimientos activistas han expuesto los mecanismos en que tales discriminaciones se materializan. En primer lugar, encontramos el ciscentrismo, que hace referencia a la concepción socialmente generalizada por la que se percibe que todas las personas poseen un género que se corresponde con el asignado en el momento del nacimiento por motivos biológicos.

Esta concepción sustenta las actitudes y juicios de valor que colocan a las personas trans en posiciones subordinadas respecto a las personas cis en el status quo, generando exclusiones y violencias de tipo material y simbólico (Radi, 2020).

En segundo lugar, en nuestra sociedad todavía persiste un enfoque mayoritario limitado a las posibilidades de género binarias (hombre y mujer) como únicas opciones existentes, lo que recibe el nombre de binarismo de género (Mateos, 2017).

En tercer lugar, el término monosexualidad es empleado para señalar las imposiciones sociales que constriñen la posibilidad de pertenencia a una sola categoría binaria: o se es hombre o se es mujer. Por último, la transfobia se constituye como otro de los mecanismos en que las discriminaciones sociales atraviesan los cuerpos que extrapolan las fronteras del género. Esta se puede definir como "diferentes formas de violencia, discriminación, odio, rechazo, agresividad y actitudes negativas sobre quienes transgreden o no encajan en las expectativas sociales sobre las normas de género" (Platero, 2014, pp. 211-212).

A la luz de los conceptos esbozados, nos planteamos los siguientes objetivos de investigación:

- Estudiar en profundidad cuál es la presencia y el abordaje de las identidades trans en los talleres de educación afectivo-sexual impartidos en la Educación Secundaria Obligatoria (ESO) en dos Institutos de Educación Secundaria (IES).

- Analizar e identificar la posible presencia de valores socioculturales y formas de discriminación hacia las identidades trans (binarismo, monosexualidad y ciscentrismo) que son reproducidos en los talleres de educación afectivo-sexual implementados en la ESO en dos IES.

\section{Metodología}

Este estudio ha sido desarrollado por dos personas investigadoras externas a los centros educativos que constituyen los casos, conformando un proceso de trabajo conjunto en las distintas etapas y momentos de la investigación, a excepción de la recogida de datos, de la que se encargó exclusivamente una de ellas, a fin de preservar un enfoque más distanciado de los casos y sus personas durante el proceso de análisis.

Los objetivos de investigación han sido abordados desde el paradigma cualitativo (Sandín, 2010), dado que este busca ge- nerar conceptos, conocimientos y comprensiones profundas y científicas de los casos estudiados desde su vivencia y las miradas de las personas involucradas, logrando una información más profunda y situada en la realidad del contexto (Stake, 1998).

Como técnica de diseño metodológico se empleó el estudio de caso múltiple. Esta estrategia de investigación permite conocer las especificidades de los casos estudiados, tanto de manera singular como en común, a la par que favorecer la transferencia de los resultados alcanzados a otros casos externos, según el grado de similitud con los estudiados, y modificar generalizaciones de otros trabajos (Stake, 1998).

\subsection{Participantes}

Los casos participantes de esta investigación atendieron a tres criterios de selección: 1) que fuesen centros de titularidad pública; 2) que guardasen un alto nivel de compromiso con el programa analizado; 3) que contasen con una amplia trayectoria en la implementación de actividades de educación afectivo-sexual en el marco del programa.

El Caso 1 atiende a alumnado de familias cuyo nivel sociocultural es catalogado como medio-bajo. Este proviene de distintas zonas de la ciudad que conforman grandes diferencias entre sí: algunas de ellas se caracterizan por tener un nivel socioeconómico alto, mientras que otras están consideradas por la administración pública como Zonas con Necesidades de Transformación Social (en adelante, ZNTS). Este centro viene desarrollando Forma Joven desde 2004, mediante la realización de talleres de una hora de duración por cada grupo durante los meses de diciembre a mayo, variando su frecuencia entre semanal y quincenal según la disponibilidad de la enfermera del centro de salud del barrio que los impartía, quien contaba con una experiencia de 5 años en el desarrollo del Programa en este instituto. En dichos talleres se abordaba la educación afectivo-sexual con $2^{\circ}$, $3^{\circ}$ y $4^{\circ}$ de ESO, así como Formación Profesional Básica (FPB).

El Caso 2 se encuentra situado en un barrio periférico almeriense declarado por la administración como ZNTS, por lo que la mayoría del alumnado proviene de entornos familiares de bajo nivel socioeconómico. Este centro viene implementando Forma Joven desde sus inicios (año 2001), con las líneas de $3^{\circ}$ y $4^{\underline{0}}$ de ESO mediante talleres de una hora de duración desde febrero hasta abril, con una periodicidad quincenal. Estos eran realizados por una médica y una enfermera del centro de salud del barrio, existiendo disparidad en cuanto a la experiencia en el Programa: la primera participa desde los inicios, mientras que la segunda no contaba con experiencia alguna, siendo los primeros años de participación.

En estos dos casos se contó con la coordinación del Programa del Equipo Directivo y el profesorado coordinador del centro.

Tabla 1.

Contenidos trabajados en el marco del Programa.

\begin{tabular}{|c|c|c|}
\hline & Curso & Contenidos \\
\hline \multirow{3}{*}{ Caso 1} & $2^{\circ} \mathrm{ESO}$ & Concepto de sexualidad; mitos y verdades. \\
\hline & $\begin{array}{c}3^{\circ} \text { ESO y } \\
\text { FPB }\end{array}$ & Importancia y forma de actuación de los métodos anticonceptivos; cómo emplearlos. \\
\hline & $4^{\circ} \mathrm{ESO}$ & Qué son las Infecciones de Transmisión Sexual (ITS); consecuencias de estas en la salud; formas de contagio; métodos para evitarlas. \\
\hline \multirow[t]{2}{*}{ Caso 2} & $3^{\circ} \mathrm{ESO}$ & $\begin{array}{l}\text { Concepto de sexualidad; categorías de sexo y género; aspectos biológicos y culturales; Derechos Humanos en torno a la sexualidad; } \\
\text { importancia, forma de actuación y uso de los métodos anticonceptivos. }\end{array}$ \\
\hline & $4^{\mathrm{o}} \mathrm{ESO}$ & Resolución de conflictos en las relaciones afectivo-sexuales; mito del amor romántico y toxicidad en la pareja; violencia de género. \\
\hline
\end{tabular}

Fuente: elaboración propia. 
En el estudio participaron 117 estudiantes ( $\mathrm{n}=83$ Caso $1 ; \mathrm{n}=34$ Caso 2), 11 profesores tutores ( $\mathrm{n}=6$ Caso $1 ; n=5$ Caso 2 ), 3 sanitarios ( $\mathrm{n}=1$ Caso $1 ; \mathrm{n}=2$ Caso 2 ), 3 coordinadores ( $\mathrm{n}=2$ Caso $1 ; \mathrm{n}=1$ Caso 2) y 2 directores ( $\mathrm{n}=1$ Caso $1 ; n=1$ Caso 2 ). Prácticamente la totalidad se correspondía con una identidad de género cis $(\mathrm{n}=135)$, mientras que una alumna del Caso 1 se correspondía con una identidad de género trans.

\subsection{Temporalización}

El trabajo de investigación ha seguido la siguiente temporalización (ver Tabla 2).

Tabla 2.

Secuenciación cronológica de la investigación.

\begin{tabular}{|c|c|c|c|c|c|}
\hline$\frac{1^{0} \text { año: }}{2014 / 15}$ & $\frac{\underline{2}^{0} \text { año: }}{2015 / 16}$ & $\frac{3^{3^{0}} \text { año: }}{2016 / 17}$ & $\frac{4^{0} \text { año: }}{2017 / 18}$ & $\frac{5^{\circ} \text { año: }}{2018 / 19}$ & $\frac{6^{0} \text { año: }}{2019 / 20}$ \\
\hline $\begin{array}{l}\text { Revisión bibliográfica y del estado } \\
\text { de la cuestión. } \\
\text { Elaboración del proyecto de } \\
\text { investigación. } \\
\text { Conocimiento a fondo de la } \\
\text { documentación del Programa. } \\
\text { Selección y primeras negociaciones } \\
\text { con los centros participantes. } \\
\text { Recogida de datos Caso } 1 .\end{array}$ & $\begin{array}{l}\text { Recogida de datos } \\
\text { Caso } 1 . \\
\text { Recogida de datos } \\
\text { Caso } 2 \text {. } \\
\text { Análisis de datos } \\
\text { (durante su recogida). } \\
\text { Revisión bibliográfica. }\end{array}$ & $\begin{array}{l}\text { Recogida de datos } \\
\text { Caso } 1 . \\
\text { Recogida de datos } \\
\text { Caso } 2 \text {. } \\
\text { Transcripción de las } \\
\text { entrevistas. } \\
\text { Análisis intensivo } \\
\text { de datos. } \\
\text { Revisión } \\
\text { bibliográfica. }\end{array}$ & $\begin{array}{l}\text { Análisis intensivo } \\
\text { de datos } \\
\text { Revisión } \\
\text { bibliográfica. }\end{array}$ & $\begin{array}{l}\text { Análisis intensivo } \\
\text { de datos. } \\
\text { Elaboración } \\
\text { informe. } \\
\text { Revisión } \\
\text { bibliográfica } \\
\text { y estado de la } \\
\text { cuestión. }\end{array}$ & $\begin{array}{l}\text { Negociación de } \\
\text { los informes. } \\
\text { Elaboración de } \\
\text { los informes } \\
\text { finales. }\end{array}$ \\
\hline
\end{tabular}

Fuente: elaboración propia.

\subsection{Técnicas de recogida de datos}

En los talleres de educación afectivo-sexual del citado programa se llevaron a cabo un total de 23 observaciones no participantes: 11 en el Caso 1 y 12 en el Caso 2. También se realizaron 51 entrevistas semi-estructuradas, 38 de ellas de carácter grupal (por grupos naturales) y 13 de carácter individual, atendiendo a todos los sectores de la comunidad educativa que participaron en el Programa (estudiantes, profesorado tutor, coordinadores, personal sanitario y directores). Por último, se analizaron 16 fuentes de tipo documental, 8 del Caso 1 y 8 del Caso 2, que fueron utilizadas como recursos didácticos en el desarrollo de los talleres: 7 diapositivas, elaboradas por el personal sanitario; 3 folletos del propio Programa Forma Joven; 6 recursos audiovisuales de fuentes televisivas, asociaciones y administraciones gubernamentales.

Se elaboraron distintas preguntas estructurales empleadas en las observaciones de los talleres de cada curso en los diferentes centros, así como en las entrevistas a los distintos sectores implicados (alumnado, profesorado tutor, profesorado coordinador, personal sanitario y Dirección de los centros). Algunas han sido conformadas a priori al trabajo de campo, concretamente a partir de los aportes recogidos en la literatura consultada, mientras que otras han surgido a medida que se recogían y analizaban los datos de la investigación.

\subsection{Análisis de los datos}

El sistema de categorías diseñado para el tratamiento de los datos ha permitido realizar un análisis temático, o de contenido, secuenciado en varias fases (ver Tabla 3). En la primera fase de análisis fue realizada una codificación y categorización inicial de forma independiente que, posteriormente, fue revisada por otros investigadores externos para garantizar una correcta aplicación del sistema de categorías diseñado. Cabe subrayar que este sistema de categorías fue fruto de un proceso de conocimiento profundo de las discriminaciones expuestas en la bibliografía consultada sobre la temática, así como de la negociación entre investigadores y personas participantes. A fin de garantizar la calidad del estudio, se emplearon distintas técnicas para asegurar la veracidad, tales como la triangulación entre los investigadores, debatiendo y contrastando las percepciones, comprensiones,

Tabla 3.

Sistemas de categorías empleado en el análisis.

Categorías (C)

C1-Ciscentrismo

C2-Binarismo

C3-Monosexualidad

\section{Subcategorías (S)}

S1.1-Exclusión o tratamiento superficial de las identidades trans entre los contenidos, S1.2-Determinar la categorización de género por factores biológicos, S1.3-Concepción biologicista de las identidades trans, S1.4-Asignar la capacidad gestante exclusivamente a las mujeres, S1.5-Ligar características biológicas al género, S1.6-Reducir la capacidad gestante únicamente a parejas heterosexuales.

S2.1- Mostrar categorías binarias de género como únicas opciones existentes, S2.2-Limitar las posibilidades de transición a Mujer a Hombre y Hombre a Mujer.

S3.1-Transición de género limitada a una categoría, S3.2-Limitar las posibilidades de género a una de las dos categorías binarias.

Fuente: elaboración propia. 
resultados e interpretaciones alcanzadas durante el proceso de análisis; la triangulación de datos, buscando la coherencia entre los resultados a partir de la comparación de la información recogida; la triangulación metodológica, mediante el contraste de la información obtenida a partir de las distintas técnicas de recogida de datos; y la validación del respondiente, por la que los resultados alcanzados fueron expuestos y debatidos con las personas participantes con el propósito de analizar su credibilidad y adecuación a la realidad (Sandín, 2000, 2010; Simons, 2011).

La participación fue voluntaria y siguió las reglas de consentimiento informado, que restringe el uso de la información únicamente a fines de investigación, asegurando el anonimato y la confidencialidad de las personas participantes.

\section{Resultados}

\subsection{Ciscentrismo}

En lo referente a la subcategoría de análisis S1.1-Exclusión o tratamiento superficial de las identidades trans entre los contenidos, los resultados hallados evidencian que las identidades trans solamente fueron trabajadas en un taller de uno de los cursos participantes en el Caso 1, descartándose su tratamiento en el resto, mientras que en el Caso 2 no se atendieron en ningún momento.

El tratamiento en este primer caso se limitó al plano puramente conceptual (explicar qué son), sin abordarlas desde una perspectiva integral y profunda (dimensión sexual, afectiva, emocional, crítica, social...). Durante su entrevista, la sanitaria afirmó que dedicó poco tiempo a la transexualidad (únicamente en los talleres de $2^{\circ}$ de ESO), centrándose en explicar que la transexualidad es algo que existe y que es normal. Igualmente, afirmó que estas identidades no aparecieron en los ejemplos, casos e imágenes que se expusieron por los siguientes motivos:

1) menor repercusión frente al resto de contenidos, dado que incumbe a un sector minoritario del alumnado y no a la totalidad de este:

El porcentaje es muy bajo. Entonces, tienen que conocer que está ahi, que es normal, pero que hay que... En fin, que le dedico poco tiempo, porque también la repercusión a nivel general pues es mucho menor. (Entrevista sanitaria. Caso 1)

2) el reducido tiempo disponible para el desarrollo del Programa, argumentando que si ampliase el trabajo sobre las identidades trans debería restar tiempo a otros contenidos que considera más importantes y que sí afectan a todo el alumnado, tales como Infecciones de Transmisión Sexual (ITS) y embarazos no deseados.

Resulta relevante que el alumnado entrevistado no mencionase las identidades trans cuando se le consultó por los contenidos aprendidos, trabajados o que les pudieran ser de utilidad de los talleres, excepto un alumno que indicó que se trató la diferencia entre transexual y homosexual. Ello puede deberse a que el alumnado percibía las identidades trans como contenido de poca importancia y de insuficiente trabajo por parte del Programa. Respecto a ello, una alumna trans del centro mostró su disconformidad hacia esta falta de abordaje. Como argumento, aportó que le gustaría que se trabajasen las identidades trans en igual medida que el resto de contenidos, ya que las dudas o problemas sobre ITS y anticoncepción pueden ser resueltos con facilidad a través de una visita al médico, mientras que las referidas a las identidades trans no disponen de una solución tan sencilla ni el alumnado encuentra los medios necesarios a su alcance.
Complementariamente, en la educación afectivo-sexual implementada en ambos institutos participantes también se transmitieron concepciones ciscentristas, dado que:

1) en relación con la subcategoría de análisis S1.2-Determinar la categorización de género por factores biológicos, se determinó el género de las personas por los órganos sexuales:

Un feto no se sabe si será niño o niña hasta un determinado momento en que se queda el clítoris, en el caso de las niñas, o se alarga y se produce el pene, en el caso de los niños. (Médica. Observación taller, $3^{\circ} \mathrm{A}$. Caso 2 )

2) en relación con la subcategoría de análisis S1.5-Ligar características biológicas al género, se transmitió que son los hombres quienes poseen pene y testículos, mientras que las mujeres tienen vagina, clítoris, útero, trompas, ovarios, labios e himen:

El himen es una membrana fina elástica que en algunas mujeres se rompe sin haber realizado un contacto sexual y en otras puede continuar después de la introducción de un dedo o un pene. No es necesario que la rotura de himen vaya acompañada de sangre, quizá sólo unas gotitas en algunas mujeres. En el caso del chico aún persiste en algunos entornos la idea de que también se le tiene que partir o romper algo, en este caso el frenillo, siendo totalmente incierto. (Diapositivas talleres, $2^{\circ}$ ESO. Caso 1)

Excitación sexual: Chicos: erección del pene y elevación de los testículos; Chicas: lubricación, erección del clitoris, aumento tamaño labios mayores y menores. (Diapositivas talleres, $2^{\circ}$ ESO. Caso 1).

En los talleres del Caso 2 encontramos, además, que se ligaron los órganos sexuales y las características anatómicas al género de forma explícita al realizar una actividad donde se pedía al alumnado que clasificara una serie de imágenes de órganos corporales según fuesen "de hombre o de mujer".

3) en relación con la subcategoría de análisis S1.3-Concepción biologicista de las identidades trans, se hizo referencia al género de las personas transexuales como "lo que sienten" en lugar de "lo que son", no respetando así su identidad autodeterminada y prevaleciendo una mirada del género ligada a las características fisiológicas:

Hay hombres que se sienten mujer y mujeres que se sienten hombres. (Sanitaria. Observación taller, $2^{\circ} \mathrm{C}$. Caso 1 )

4) en relación con la subcategoría de análisis S1.4-Asignar la capacidad gestante exclusivamente a las mujeres, se ligó la capacidad de embarazo a estas, excluyendo a los hombres trans preoperados/prehormonados que pueden quedar embarazados y a las mujeres trans que no tienen posibilidad de ello:

Esas células sirven para dejar embarazada a una chica. (Médica. Observación taller, $3^{\circ} \mathrm{A}$. Caso 2)

La sanitaria comenta que es importante tener una buena autoestima, por ejemplo, a la hora de ponerte el preservativo para tener relaciones sexuales. En el caso de la mujer para evitar embarazos y en el caso del hombre enfermedades de transmisión sexual. (Sanitaria. Observación taller, $2^{\circ} \mathrm{A}$. Caso 1 )

5) en relación con la subcategoría de análisis S1.5-Ligar características biológicas al género, se afirmó que existen diferencias fisiológicas entre hombres y mujeres, así como que la menstrua- 
ción es propia de las mujeres o que los hombres tienen la capacidad de producir esperma, se explicaron los síntomas de las ITS en función de los órganos y se relacionaron con el género, y se asignaron los diferentes tipos de métodos anticonceptivos a ser hombre o mujer:

Hombres y mujeres somos iguales en derechos, pero somos distintos físicamente. (Médica. Observación taller, 3ํA. Caso 2).

La médica pregunta el por qué le llaman «huevos» y explica que de ahí salen las células, óvulos, que cuando se juntan con el esperma del chico en la trompa sale el feto. (Observación taller, $3^{\circ} \mathrm{A}$. Caso 2)

La sanitaria comenta que el preservativo femenino es tan grande puesto que el tamaño de la vagina de las mujeres es muy diferente. (Observación taller, 3ํDiversificación y FPB. Caso 1)

La clamidia es una infección causada por una bacteria que afecta al cuello del útero en las mujeres, y a la uretra en los hombres (Diapositivas talleres, $4^{\circ}$ ESO. Caso 1 )

En uno de los vídeos empleado como recurso didáctico en los talleres del Caso 2 se expuso una silueta con los patrones tradicionalmente asignados al modelo tradicional de mujer (cuerpo curvado, pechos desarrollados y pelo largo con corte "femenino") para explicar la menstruación, transmitiendo una concepción parcializada de la capacidad de ovular que excluye a los hombres trans preoperados/prehormonados. Este ciscentrismo se acrecentó al repetirse el empleo de siluetas con características tradicionales de mujeres con órganos genitales de la categoría de sexo hembra para explicar la píldora anticonceptiva, el anillo vaginal y el parche anticonceptivo.

Asimismo, no se rebatieron las concepciones ciscentristas del alumnado, que relacionó las características genitales al género:

La médica pregunta que dónde está el órgano sexual de la chica y un alumno dice que es el clítoris. La médica pregunta si está fuera o dentro de la vagina, respondiendo un alumno que fuera (Observación taller, $3^{\circ} \mathrm{C}$. Caso 2 )

6) en relación con la subcategoría de análisis S1.6-Reducir la capacidad gestante únicamente a parejas heterosexuales, se mostró la posibilidad de embarazo como algo propio de relaciones conformadas por un hombre y una mujer, sin tener en cuenta que existen parejas del mismo género con capacidad gestante si una de las personas es trans preoperada/prehormonada:

La médica comenta que dos hombres y dos mujeres pueden realizar sexo por placer, pero no para reproducción porque no se pueden reproducir (Observación taller, $3^{\circ} \mathrm{B}$. Caso 2 )

Un amplio número del alumnado entrevistado manifestó haber adquirido o trabajado en los talleres de educación afectivo-sexual aprendizajes ciscentristas, afirmando que: se transmitió que las chicas tienen el placer "por fuera" y los chicos "por dentro"; se expuso un dibujo donde se mostraban las diferencias anatómicas entre hombres (asignándole el pene como órgano sexual) y las mujeres (pechos, clítoris y vagina); se ligó el preservativo vaginal a las mujeres; se mostró como imposible la inseminación natural en relaciones entre dos mujeres; se relacionó el daño en el himen en la "primera vez" a las mujeres; se determinó la menstruación a las mujeres; y se asignó la eyaculación de semen a los hombres. Estas manifestaciones de ciscentrismo que señaló el alumnado coinciden con las percibidas en el análisis de los talleres estudiados.
A modo general, también se evidenció que las identidades trans no se conformaban como una temática presente a abordar desde las miradas de quienes participaban en la coordinación e implementación de la educación afectivo-sexual en nuestros casos (profesorado coordinador, Dirección y personal sanitario) al no ser contempladas como contenidos $u$ objetivos de este, a la vez que reproducían muchos valores excluyentes y discriminatorios. Ello puede suponer un elemento que impedía la reflexión de las prácticas llevadas a cabo sobre la presencia y tratamiento de la diversidad de género. Mientras tanto, el personal encargado de la organización e implementación de los talleres de educación afectivo-sexual, así como el profesorado tutor de los cursos participantes, mantenía una visión de la educación afectivo-sexual más ligada a un modelo prevencionista y sanitario debido a cuatro factores principales, según las personas informantes: 1) la medicalización de la pedagogía de la sexualidad;2) que los propósitos iniciales del Programa sobre sexualidad se centraban en la prevención de ITS y embarazos no deseados, pudiendo suponer una dificultad añadida a la hora de tratar otros contenidos; 3) considerarse el colectivo LGTBI un sector minoritario y, por tanto, de menor repercusión educativa; 4) el desconocimiento de este personal sanitario y educativo sobre las formas de identidad de género no-hegemónicas.

\subsection{Binarismo}

Los resultados sobre la subcategoría de análisis S2.1-Mostrar categorías binarias de género como únicas opciones existentes, reflejaron que, en el Caso 1, se cayó en una mirada binarista en su abordaje, transmitiendo las categorías hombre y mujer como únicas posibilidades de transición de género:

Identidad sexual es sentirse hombre o sentirse mujer, no tiene porqué ir ligado al sexo biológico. (Enfermera. Observación taller, 2ํ․ Caso 1 )

En los talleres realizados en este instituto también encontramos que otras formas de género no binarias no estaban presentes en el discurso de la sanitaria que los impartía, reflejándose en la concepción que el alumnado mantenía del Programa:

-Leo ${ }^{1}$ : Dijeron que había hombres que se sentían mujeres...

-Jesús: Y mujeres que se sentían hombres. (Entrevista alumnado, $2^{\circ}$ B. Caso 1 )

En relación con la subcategoría de análisis S.2.1-Limitar las posibilidades de transición a Mujer a Hombre y Hombre a Mujer, en los talleres llevados a cabo en el Caso 1 se mostró una visión de las transiciones de género reducida a las binarias:

Explica también que puede ser transexual mujer u hombre, pero lo normal es que sean hombres que se cambien a mujeres. (Observación taller, $2^{\circ} \mathrm{A}$. Caso 1 )

\subsection{Monosexualidad}

Atendiendo a la subcategoría de análisis S3.1-Transición de género limitada a una categoría, hallamos que, cuando se trabajaron las identidades trans, se transmitió como única posibilidad la transición a una de las dos categorías hegemónicas, tal y como evidencia el último fragmento de observación expuesto en los resultados sobre binarismo.

Tal y como se refleja en ese mismo fragmento, así como en el que se expone a continuación, el análisis de los datos también

Los nombres de las personas participantes que aparecen en este trabajo son ficticios a fin de preservar el anonimato. 
ofrece resultados sobre la subcategoría S3.2-Limitar las posibilidades de género a una de las dos categorías binarias, al transmitirse que las personas solamente podemos pertenecer a una de las dos identidades de género hegemónicas:

Pregunta al alumnado: ¿los hombres tienen más deseo sexual que las mujeres? El alumnado dice que falso y la sanitaria dice que efectivamente, "no depende tanto de ser hombre o mujer, sino de cada persona». (Observación taller, $2^{\circ} \mathrm{B}$. Caso 1 )

\section{Discusión y conclusiones}

En un mundo donde las manifestaciones de la sexualidad, así como las formas de vivirla, transitarla y manifestarla pueden parecer más complejas por la diversidad emergente visibilizada, no puede abordarse todavía una educación afectivo-sexual en clave de simplicidad. La sexualidad debe ser atendida desde una educación integral, democrática, profunda e igualitaria para educar "en, desde y para" la diversidad, a fin de no reproducir valores antagónicos a la igualdad de derechos y oportunidades del colectivo LGTBI. No obstante, tal y como demuestran los resultados alcanzados, la presencia de concepciones excluyentes, la transmisión de los valores discriminatorios, así como el silenciamiento y el tratamiento superficial del colectivo de personas trans se constituyen como realidades en las prácticas de educación afectivo-sexual estudiadas.

Otras investigaciones (Jones y Hillier, 2012; Ortmann, 2011; Sherlock, 2015) también mostraron cómo, desde los modelos educativos en general y más concretamente desde el ámbito de la educación afectivo-sexual, las orientaciones, identidades y corporalidades LGTBI sufren la invisibilización y silenciamiento por su omisión entre los contenidos abordados al considerarse políticamente incorrectos desde la mirada social imperante. En consonancia, las personas LGTBI participantes de las investigaciones de Hobaica y Kwon (2017), Rivers y Noret (2008) y Smith et al. (2008) coincidían en que la educación afectivo-sexual recibida les generaba sentimientos excluyentes, tales como sentirse invisibles, sexualmente desprevenidas o avergonzadas. Ante ello, las prácticas de educación afectivo-sexual carecen de utilidad para este alumnado por el hecho de no atender sus realidades (Estes, 2016), no encontrando más opción que recurrir a vías de educación informal (internet, pornografía, medios de comunicación...) para satisfacer los deseos y necesidades de aprendizaje sobre sexualidad que quedan sin atender desde las instituciones educativas (Currin et al., 2017).

Retomando los resultados obtenidos, podemos afirmar que no se concebía la educación afectivo-sexual como un espacio para el abordaje de las identidades de género no-hegemónicas. Al igual que los datos arrojados por De Almeida (2015), se partía de un enfoque tradicional de la salud para centrarse en los riesgos sexuales, tales como las ITS y los embarazos no deseados. Así, resulta necesario repensar cuáles son los propósitos a alcanzar y, por ende, qué presencia y tratamiento han de tener las sexualidades minoritarias en este campo educativo. $Y$ es que no debemos olvidar que el campo de la educación afectivo-sexual se presenta como el espacio educativo con mayor potencialidad para trabajar hacia la igualdad de derechos y oportunidades en lo referido a sexualidades, identidades de género y corporalidades (Epstein y Johnson, 2000; Ortmann, 2011).

En esta línea, atendiendo a las aportaciones de Sánchez (2009), la omisión de las formas no normativas de ser, sentir, vivir y transitar la sexualidad, así como la existencia de valores discriminatorios en los talleres educativos, reproduce y legitima la "normalización" y "naturalización" de las identidades de gé- nero hegemónicas, prevaleciendo estas como dominantes en el status quo. Del mismo modo, la reproducción de la percepción de la sexualidad limitada a la normatividad contribuye a la clasificación, jerarquización y disciplinamiento de las identidades y los cuerpos. Todo ello, legitima y genera en el alumnado la conformación de subjetividades naturalizadas en el desempeño de exclusiones, discriminaciones y opresiones contra las identidades y corporalidades no-hegemónicas, contribuyendo a su censura social.

Resulta imprescindible por tanto evitar el ciscentrismo desde la educación afectivo-sexual: no ligar características fisiológicas al género con el objetivo de no excluir a las personas trans en nuestro discurso, así como hacerlas presentes entre los contenidos abordados a través de una formación integral de la sexualidad (sexo, afectividad, amor, relaciones y derechos). Dicha presencia no debe reducirse a momentos puntuales y aislados, ni al discurso de la tolerancia, pues se contribuye a la percepción de las sexualidades no hegemónicas como "lo otro" (Louro, 2018).

Igualmente, se ha de atender al cuidado del contenido de los mensajes transferidos, en cuanto que estos se establecen como canales de transmisión de valores que conforman los idearios del alumnado. Valores que pueden reproducir concepciones excluyentes $\mathrm{o}$, por el contrario, igualitarias y que, a menudo, se transmiten de manera sutil e inconsciente debido a las concepciones y valores interiorizados por la persona comunicante. Por tanto, cambiar los paradigmas dominantes, tan a menudo presentes entre el personal docente y que son transmitidos al alumnado de manera imperceptible, es de vital importancia dada su amplia presencia en la normatividad sociocultural. En esta línea, los resultados evidencian que las exclusiones y discriminaciones hacia las identidades trans no se debían a una intencionalidad hiriente del personal educativo y sanitario, sino a una carencia de conocimientos que provocaba la reproducción de mensajes y percepciones no igualitarias. Así, los hallazgos de esta investigación subrayan la necesidad de incorporar el abordaje de las identidades y corporalidades en la formación universitaria de cara a proporcionar un amplio abanico de conocimientos y valores que no reproduzcan discriminaciones en los contextos educativos (Gavilán, 2016; Jones et al., 2014; Platero, 2014).

Del mismo modo, exponer marcos de referencia para que las personas trans conozcan experiencias que rompan con las concepciones, valores y actitudes socialmente dominantes conforma una vía útil en el camino hacia un abordaje inclusivo e igualitario. En consecuencia, pueden ser trabajados contenidos que permitan la adquisición de conocimientos y actitudes sobre la existencia de personas que no se ajustan a los modelos binarios y monosexuales o que rompen con la categorización de tipo biológico, así como cuerpos que exceden las posibilidades fisiológicas tradicionalmente ligadas a los géneros, como es el caso de los cuerpos gestantes masculinos. Algunos de estos casos son el del estadounidense Thomas Beatie, el primer hombre embarazado que fue documentado; el del español Rubén Noé Coronado Jiménez, en 2009; el del californiano Scott Moore; el del argentino Alexis Taborda, en 2013; el del inglés Hayden Cross; el del estadounidense Trystan Reese; el del ecuatoriano Fernando, primer hombre documentado en haber quedado embarazado de su pareja mujer, Diane; o el del español Rubén Castro. De este modo, avanzaremos para que las prácticas de educación afectivo-sexual se conformen como espacios educativos en los que, por un lado, el alumnado trans se sienta reconocido, encontrando figuras de representación de las realidades socialmente excluidas desde los contextos educativos, y por otro lado, rompamos las concepciones discriminatorias y el desconocimiento desde la visibilidad de realidades tradicionalmente invisibilizadas. 
A partir de ello, la educación afectivo-sexual puede dejar de ser un mecanismo de reproducción de exclusiones y opresiones en lo referido a la identidad de género, para convertirse en un espacio de conformación de valores democráticos, basados en la visibilización, el respeto y la valorización de la diversidad social existente, con el objetivo de que estos principios igualitarios se vean reflejados en la mirada, aceptación e inclusión de la diversidad de género en nuestra sociedad.

\section{Referencias bibliográficas}

Balza, I. (2009). Bioética de los cuerpos sexuados: transexualidad, intersexualidad y transgenerismo. Revista de Filosofía Moral y Política Isegoría, 40, 245-258. https://cutt.ly/1kE7hpu

Butler, J. (2007). El género en disputa. Paidós.

Chi, X., Hawk, S.T., Winter, S. y Meeus, W. (2015). The Effect of Comprehensive Sexual Education Program on Sexual Health Knowledge and Sexual Attitude Among College Students in Southwest China. Asia-Pacific Journal of Public Health, 27(2), 2049-2066. https://doi.org/10.1177/1010539513475655

Collignon Goribar, M.M. (2011). Discursos sociales sobre la sexualidad: narrativas sobre la diversidad sexual y prácticas de resistencia. Comunicación y sociedad, 16, 133-160. https://doi. org/10.32870/cys.v0i16.1118

Currin, J. M., Hubach, R.D., Durham, A.R., Kavanaugh, K.E., Vineyard Z. y Croff, J.M. (2017). How gay and bisexual men compensate for the lack of meaningful sex education in a socially conservative state. Sexuality, Society and Learning, 17(6), 667-681.

De Palma, R. y Francis, D.A. (2014). The gendered nature of South African teachers' discourse on sex education. Health Education Research, 29(4), 624-632. https://doi.org/10.1093/her/ cyt117

Ekstrand, M., Engblom, C., Larsson, M. y Tydén, T. (2011). Sex education in Swedish schools as described by young women. The European Journal of Contraception \& Reproductive Health Care, 16(3), 210-224.

Epstein, D. y Johnson, R. (2000). Sexualidades e institución escolar. Morata

Estes, M.L. (2016). "Well if there's one benefit, you're not going to get pregnant": A qualitative investigation of the sex education that gay, lesbian, and bisexual individuals received (Doctoral Thesis). Middle Tennessee State University, Tennessee, Estados Unidos. https://cutt.ly/qkE7QJi

Foucault, M. (2012). Historia de la sexualidad 1. La voluntad del saber. Biblioteca Nueva.

Gavilán, J. (2016). Infancia y transexualidad. Los libros de la catarata.

Grotz, E., Díaz, A., González, M.C. y Plaza M.V. (2016). La educación sexual del colectivo trans: una comparación entre el currículo real de un bachillerato popular y el prescripto en los lineamientos curriculares nacionales de Argentina. Bio-grafía. Escritos sobre la Biología y su enseñanza, 9(16), 205-222.

Hobaica, S. y Kwon, P. (2017). "This Is How You Hetero:" Sexual Minorities in Heteronormative Sex Education. American Journal of Sexuality Education, 12(4), 423-450.
Jones, T.M. y Hillier, L. (2012). Sexuality education school policy for Australian GLBTIQ students. Sex Education: Sexuality, Society and Learning, 12(4), 437-454. https://doi.org/10.1080/1468 1811.2012.677211

Jones, T., Smith, E., Ward, R., Dixon, J., Hillier, L. y Mitchell, A. (2016). School experiences of transgender and gender diverse students in Australia. Sex Education, 16(2), 156-171. https:// doi.org/10.1080/14681811.2015.1080678

Lamas, M. (2009). El fenómeno trans. Debate Feminista, 39, 3-13. https://cutt.ly/SkE7Dlf

Louro, G.L. (2018). Currículo, género y sexualidad. Lo "normal”, lo "diferente" y lo "excéntrico". Descentrada, 3(1). https://doi. org/10.24215/25457284e065

Mateos Casado, C. (2017). Binarismo. En R.L. Platero, M. Rosón y E. Ortega (Eds.). Barbarismos queer y otras esdrújulas (pp. 8288). Ediciones Bellaterra.

Morton Ninomiya, M. (2010). Sexual health education in Newfoundland and Labrador schools: Junior high school teachers' experiences, coverage of topics, comfort levels and views about professional practice. The Canadian tournai of $\mathrm{Hu}$ man Sexuality, 1, 15-26.

Ortmann, C. (2011). Desandar la sexualidad escolarizada. Ponencia presentada en XVI Congreso Pedagógico UTE, Buenos Aires, Argentina. https://cutt.ly/dkE7J7d

Platero Méndez, R.L. (2014). Trans*exualidades: acompañamiento, factores de salud y recursos educativos. Edicions Bellaterra.

Preciado, P.B. (2003). Multitudes queer: notas para una política de los "anormales". Revista Multitudes, 12. https://cutt.ly/ nkE7VmL

Quaresma da Silva, D. y Ulloa Guerra, O. (2011). Prácticas de educación sexual: un análisis en escuelas municipales del sur de Brasil. Education Policy Analysis Archives, 19, 1-23. https:// cutt.ly/gkE70rp

Radi, B. (2020). Notas (al pie) sobre cisnormatividad y feminismo. Ideas. Revista de filosofía moderna y contemporánea, 11, 2336. https://cutt.ly/smk5yUi

Rivers, I. y Noret, N. (2008). Well-being among same-sex and opposite-sex-attracted youth at school. School Psychology Review, 37(2), 174-187.

Sánchez Sáinz, M. (2009). Introducción: bases conceptuales. En M. Sánchez Sáinz (Coord.). Cómo educar en la diversidad afectivo-sexual en los centros escolares (pp. 25-30). Los Libros de la Catarata.

Sandín, M.P. (2000). Criterios de validez en la investigación cualitativa: de la objetividad a la solidaridad. Revista de Investigación Educativa, 18(1), 223-242.

Sandín, M.P. (2010). Investigación cualitativa en educación: Fundamentos y tradiciones. McGraw-Hill.

Sherlock, L. (2015). Inclusive sex educación: Narratives of good practice from Ireland and Sweden (Doctoral Thesis). Trinity College Dublin, Dublín, Irlanda. https:/cutt.ly/SkE78GJ

Simons, H. (2011). Estudio de caso: teoría y práctica. Morata.

Smith, A., Agius, P., Mitchell, A., Barrett C. y Pitts, M. (2008). Secondary Students and Sexual Health: Results of the 4th National Survey of Australian Secondary Students, HIVIAIDS and Sexual Health. Research Centre in Sex, Health and Society.

Stake, R.E. (1998). Investigación con estudio de casos. Morata. 